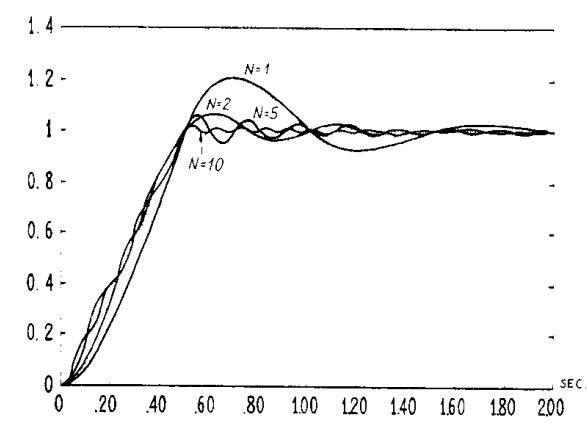

Fig. 3. Responses of multirate controlled systems to step changes in the reference signal.

TABLE I

Discrete Transfer Functions of Some Multirate Controllers

\begin{tabular}{|l|l|}
\hline $\mathrm{n}=1$ & $\mathrm{R}(\mathrm{z})=\hat{\mathrm{R}}_{1}(\hat{z})=10^{-2}\left(1.8767-0.0668 \hat{z}^{-1}\right) /\left(1+0.3567 \hat{z}^{-1}\right)$ \\
$\mathrm{n}=2$ & $\hat{\mathrm{R}}_{2}(\hat{z})=10^{-2}\left(2.598-0.49 \hat{z}^{-1}\right) /\left(1+0.58 \hat{z}^{-1}\right)$ \\
$\mathrm{n}=5$ & $\hat{\mathrm{R}}_{5}(\hat{z})=10^{-2}\left(4.9365-2.5336 \hat{z}^{-1}\right) /\left(1+0.801 \hat{z}^{-1}\right)$ \\
$\mathrm{n}=10$ & $\hat{\mathrm{R}}_{10}(\hat{z})=10^{-2}\left(8.9135-6.386 \hat{z}^{-1}\right) /\left(1+0.895 \hat{z}^{-1}\right)$ \\
\hline
\end{tabular}

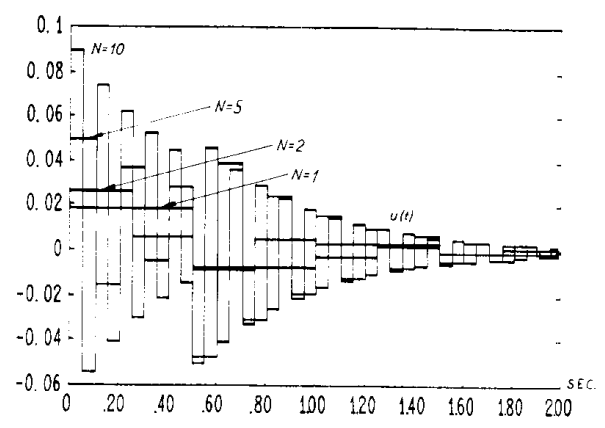

Fig. 4. Control signals generated by the multirate controllers.

The proposed controllers perform a control action that is faster than the sampling period, and allow control of the output signal between sampling instants. The example of this paper (and other examples [15]) shows that the transient response is significantly improved with only $n=2$. Higher values of $n$ normally give better results. Plots of the control signal $u(t)$ (Fig. 4) show that the control action presents two components. The first one is the classical feedback control action of period $T$ that changes as a function of the output sequence $\left\{y_{k}\right\}$. The second one is a quick "feedforward action" that changes every $T / n$ seconds. This last action controls the output signal $y(t)$ in the intervals between sampling instants.

The order of these multirate controllers is the same or slightly higher than the order of the normal controllers. So this method does not give rise to complex controllers.

Finally, it has to be mentioned that the sensitivity of the controlled system does not suffer significantly because of the use of these multirate controllers; in fact, in many cases, it is similar.

\section{REFERENCES}

[1] B. C. Kuo, Digital Control Systems. New York: Holt, Rinehart and Winston, 1980.

[2] K. S. Rattan, "Digital redesign of multiloop continuous control systems," in Proc. 1981 Joint Automat. Contr. Conf., Univ. Virginia, Charlottesville, June 1981.

[3] R. F. Whitbeck and L. G. Hofmann, "Analysis of digital flight control systems with flying qualities applications," Rep. AFFDL-TR-78-115, vol. II, Sept. 1978.

[4] R. F. Whitbeck and L. G. Hofmann, "Digital control law synthesis in the wdomain," AIAA J. Guidance Contr., vol. 1, Sept.-Oct. 1978.

[5] D. P. Glasson, "Development and applications of multirate digital control," IEEE Contr. Syst. Mag., vol. 3, pp. 2-8, Nov. 1983.
[6] K. J. Astrom and B. Wittenmark, Computer Controlled Systems. Englewood Cliffs, NJ: Prentice-Hall, 1984, p. 59.

[7] P. P. Khargonekar, K. Poola, and H. Tannenbaum, "Robust control of linear timeinvariant plants using periodic compensation," IEEE Trans. Automat. Contr., vol. AC-30, pp. 1088-1096, Nov. 1985.

[8] A. B. Chammas and C. T. Leondes, "On the finite time control of linear systems by piecewise constant output feedback," Int. J. Contr., vol. 30, pp. 227-234, 1979.

[9] P. T. Kabamba, "Control of linear systems using generalized sampled-data hold functions," IEEE Trans. Automat. Contr, vol. AC-32, pp. 772-783, Sept. 1987.

[10] A. Jiménez and R. Aracil, "Digital transfer functions computation," IEEE Trans. Syst., Man, Cybern., vol. SMC-11, pp. 440-441, 1981.

[11] R. Aracil, A. Jiménez, and V. Feliu, "Multirate sampling technique in digital control systems simulation," IEEE Trans. Syst., Man, Cybern., vol. SMC-14, pp. 776-780, Sept. 1984.

[12] H. F. VanLandinghan, Introduction to Digital Control Systems. New York: Macmillan, 1985, pp. 273-276.

[13] P. Katz, Digital Control Using Microprocessors. Englewood Cliffs, NJ: Prentice-Hall, 1982.

[14] G. C. Franklin and J. D. Powell, Digital Control of Dynamic Systems. Read ing, MA: Addison-Wesley, 1980.

[15] V. Feliu, "Reguladores multiperiódicos. Aplicación a plantas muestreadas lentamente," Ingeniería de Sistemas y Automática, Universidad Politécnica de Madrid, Spain, Int. Rep., 1986.

\title{
Decentralized Multirate Control
}

\author{
M. E. SEZER AND D. D. ŠILJAK
}

\begin{abstract}
The objective of this note is to introduce a decentralized control approach to multirate linear systems. Each control channel is assigned a single sampling rate, which provides simplicity in design of local controllers. This control strategy is natural for plants with widely different characteristic frequencies, and suitable for implementation using multiprocessor schemes.
\end{abstract}

\section{Introductron}

In controlling systems having widely different time constants, it has been customary to use multirate digital control. Since the early work of Sklansky and Ragazzini [1], Kranc [2], and Kalman and Bertram [3], a considerable number of results on multirate sampled-data control systems have been reported in the literature, as surveyed by Lu and Gupta [4] and Glasson [5]. Both frequency and state-space models have been utilized, and standard concepts of system theory have been reformulated in this context. Recently, using the Kalman-Bertram approach, Araki and Yamamoto [6] developed a state-space description of multirate systems, which provided a basis for both the transfer function and time-domain design methods and, in particular, for a solution of the pole-assignment problem by Araki and Hagiwara [7].

In this paper, we propose a decentralized approach to control of multirate systems, which is consistent with a natural grouping of input and output signals induced by their characteristic frequencies. This approach is particularly suitable for implementation of control using distributed multiprocessors operating at different sampling rates. Each processor is assigned to a group of signals having the same sampling rate, and is responsible for generating a control input based on the local information provided by the outputs of the group.

Manuscript received October 23, 1987; revised October 23, 1988. Paper recommended by Past Associate Editor, D. Castanon. This work was supported in part by the National Science Foundation under Grant ECS-8813273 and in part by the Science and Engineering Center of the Bilkent University, Turkey.

M. E. Sezer is with the Department of Electrical and Electronics Engineering, Bilkent University, Ankara, Turkey.

D. D. Siljak is with the Department of Electrical Engineering, Santa Clara University. Santa Clara, CA 95053.

IEEE Log Number 8931904 


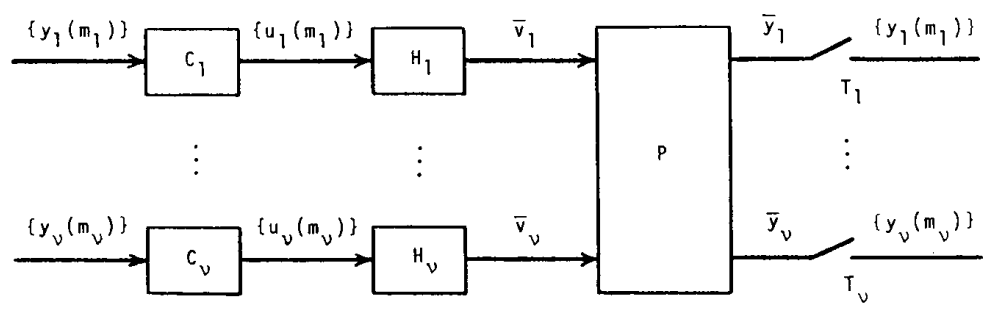

Fig. 1. Decentralized multirate sampled-data system.

The fundamental problem of decentralized control schemes has been to provide conditions under which a system can be stabilized using local controllers. The existing results for continuous-time systems of Wang and Davidson [8], Corfmat and Morse [9], and Sezer and Siljak [10] can easily be carried over to the discrete-time case. However, if a discrete system is a product of sampling a continuous plant, an interpretation of the discrete-time conditions in terms of the original plant is not straightforward, even in the case of a single-rate sampling. The situation is further complicated by multirate sampling mainly because the corresponding discrete models are either nonminimal (Araki and Yamamoto [6]) or time varying (Amit [11])

The main objective of this note is to show that the decentralized stabilizability conditions for multirate sampled-data systems are essentially the same as those for the underlying continuous-time plants. We consider only the systems with sampling rates having integral ratios, but more general cases can be considered using the same machinery.

\section{Decentralized Multrate Systems}

Consider a multirate sampled-data system with $\nu$ channels, which is shown in Fig. 1. The continuous-time plant is described by

$$
\begin{gathered}
P: \dot{\bar{x}}=\bar{A} \bar{x}+\sum_{i=1}^{\nu} \bar{B}_{i} \bar{v}_{i} \\
\bar{y}_{i}=C_{i} \bar{x}, \quad i=1,2, \cdots, \nu
\end{gathered}
$$

where $\bar{x}(t) \in \bar{\beta}^{n}, \bar{v}_{i}(t) \in \mathcal{R}^{p_{i}}$, and $\bar{y}_{i}(t) \in \bar{\beta}^{q_{i}}$ are the state, input, and output of the $i$ th channel at time $t \in \mathbb{R}$. The matrices $\bar{A}, \bar{B}_{i}, \bar{C}_{i}$ are constant and have appropriate dimensions. As is standard, we assume that $\boldsymbol{P}$ is centrally controllable and observable, but is not both controllable and observable from any individual channel.

The samplers are assumed to start at $t=0$ simultaneously, and have the sampling periods

$$
T_{i}=M_{i} \tau, \quad i=1,2, \cdots, \nu
$$

where $M_{i}$ are positive integers, and the time interval $\tau$ is the greatest common divisor of the $T_{i}$ 's, which can conveniently be regarded as a basic unit of time. The blocks $\boldsymbol{H}_{i}$ contain zero-order holds with transfer function $\left(1-e^{-T_{i} s}\right) / s$. The digital feedback controllers $C_{i}$ operate on the sampled output sequences $\left\{y_{i}\left(m_{i}\right)\right\}$ to produce the input sequences $\left\{u_{i}\left(m_{i}\right)\right\}$ to the hold circuits where $y_{i}\left(m_{i}\right)=\bar{y}_{i}\left(m_{i} T_{i}\right)$.

To obtain a discrete-time representation of the open-loop system, which consists of the plant and the hold circuits, we define the com mon sampling period $T$ as

$$
T=M \tau
$$

where $M$ is the least common multiple of the $M_{i}$ 's. We also define the discrete-time signals

$$
\begin{gathered}
x(l, m)=\bar{x}\left[(l T+m \tau)^{+}\right] \\
v_{i}(l, m)=\bar{v}_{i}\left[(l T+m \tau)^{-}\right], \quad i=1,2, \cdots, \nu
\end{gathered}
$$

for $l=0,1, \cdots$, and $m=0,1, \cdots, M-1$, and the switching functions

$$
\Delta_{i}(m)=\left\{\begin{array}{ll}
1, & m=k M_{i} \\
0, & m \neq k M_{i}
\end{array} \quad i=1,2, \cdots, \nu .\right.
$$

From $(2.1)$, we obtain

$x(l, m+1)=A x(l, m)$

$$
+\sum_{i=1}^{\nu}\left\{\Delta_{i}(m) B_{i} u_{i}(l, m)+\left[1-\Delta_{i}(m)\right] B_{i} v_{i}(l, m)\right\}
$$

and

$v_{i}(l, m+1)=\left[1-\Delta_{i}(m)\right] v_{i}(l, m)+\Delta_{i}(m) u_{i}(l, m)$,

$$
i=1,2, \cdots, \nu
$$

where

$$
A=\exp (\tau \bar{A}), \quad B_{i}=\int_{0}^{\tau} \exp (t \bar{A}) \bar{B}_{i} d t
$$

and the double-indexed sequence $\left\{u_{i}(l, m)\right\}$ is defined by

$$
u_{i}(l, m)= \begin{cases}0, & m \neq k M_{i} \\ u_{i}\left(l \frac{M}{M_{i}}+k\right), & k=0,1, \cdots, \frac{M}{M_{i}}-1 ; m=k M_{i} .\end{cases}
$$

By defining $\left\{y_{i}(l, m)\right\}$ similarly, and letting

$$
\begin{gathered}
v(l, m)=\left[v_{1}^{T}(l, m), v_{2}^{T}(l, m), \cdots, v_{\nu}^{T}(l, m)\right]^{T} \\
u(l, m)=\left[u_{1}^{T}(l, m), u_{2}^{T}(l, m), \cdots, u_{\nu}^{T}(l, m)\right]^{T} \\
y(l, m)=\left[y_{1}^{T}(l, m), y_{2}^{T}(l, m), \cdots, y_{\nu}^{T}(l, m)\right]^{T},
\end{gathered}
$$

we obtain a discrete-time representation of the open-loop system

$S:\left[\begin{array}{l}x(l, m+1) \\ v(l, m+1)\end{array}\right]$

$$
\begin{gathered}
=\left[\begin{array}{ll}
A & B[I-\Delta(m)] \\
0 & I-\Delta(m)
\end{array}\right]\left[\begin{array}{l}
x(l, m) \\
v(l, m)
\end{array}\right]+\left[\begin{array}{l}
B \Delta(m) \\
\Delta(m)
\end{array}\right] u(l, m) \\
y(l, m)=\Delta(m) C x(l, m)
\end{gathered}
$$

where

$$
\begin{gathered}
B=\left[\begin{array}{cc}
B_{1} & B_{2} \cdots B_{\nu}
\end{array}\right] \\
C=\left[\begin{array}{ll}
C_{1}^{T} & C_{2}^{T} \cdots C_{\nu}^{T}
\end{array}\right]^{T} \\
\Delta(m)=\operatorname{diag}\left\{\Delta_{1}(m) I_{1}, \Delta_{2}(m) I_{2}, \cdots, \Delta_{\nu}(m) I_{\nu}\right\}
\end{gathered}
$$

and $I_{i}$ are identity matrices of appropriate dimensions. It should be understood that in $(2.11), x(l, M)=x(l+1,0)$ and, likewise, for other signals $(2.10)$. We note that $(2.11)$ represents a periodically time-varying system with period $M$.

The decentralized digital controllers are linear, time-invariant systems represented by

$$
\begin{gathered}
C_{i}: z_{i}\left(m_{i}+1\right)=F_{i} z_{i}\left(m_{i}\right)+G_{i} y_{i}\left(m_{i}\right) \\
u_{i}\left(m_{i}\right)=H_{i} z_{i}\left(m_{i}\right)+K_{i} y_{i}\left(m_{i}\right), \quad i=1,2, \cdots, \nu
\end{gathered}
$$


where $z_{i}\left(m_{i}\right) \in \Re^{i}$ and $F_{i}, G_{i}, H_{i}, K_{i}$ are constant matrices of appropriate dimensions. In terms of double-indexed sequences, $C_{i}$ can conveniently be represented as

$$
\begin{aligned}
& C_{i}: z_{i}(l, m+1)=\left[I_{i}+\Delta_{i}(m)\left(F_{i}-I_{i}\right)\right] z_{i}(l, m)+G_{i} y_{i}(l, m) \\
& u_{i}(l, m)=\Delta_{i}(m) H_{i} z_{i}(l, m)+K_{i} y_{i}(l, m), \quad i=1,2, \cdots, \nu .
\end{aligned}
$$

Obviously, when $\boldsymbol{C}_{i}$ is a static controller, (2.14) reduces to a feedback control law

$$
u_{i}(l, m)=K_{i} y_{i}(l, m) .
$$

The collection (2.14) of controllers $C_{i}$ can be put in a compact form:

$$
\begin{gathered}
C: z(l, m+1)=[I+(F-I) \Delta(m)] z(l, m)+G y(l, m) \\
u(l, m)=\Delta(m) H z(l, m)+K y(l, m)
\end{gathered}
$$

where $z(l, m)=\left[z_{1}^{T}(l, m), z_{2}^{T}(l, m), \cdots, z_{\nu}^{T}(l, m)\right]^{T}$ and $F, G, H, K$ are block diagonal matrices with blocks $F_{i}, G_{i}, H_{i}, K_{i}$

The closed-loop system composed of $S$ and $C$ has the representation

$$
\begin{gathered}
\hat{s^{\tau}}: \xi(l, m+1)=\Phi(m) \xi(l, m) \\
y(l, m)=\Psi(m) \xi(l, m)
\end{gathered}
$$

where $\xi(l, m)=\left[x^{T}(l, m), z^{T}(l, m), v^{T}(l, m)\right]^{T}$ and

$$
\Phi(m)=\left[\begin{array}{ccc}
A+B \Delta(m) K C & B \Delta(m) H & B[I-\Delta(m)] \\
G \Delta(m) C & I+(F-I) \Delta(m) & 0 \\
\Delta(m) K C & \Delta(m) H & I-\Delta(m)
\end{array}\right]
$$

The transitions of $\hat{\boldsymbol{S}^{\tau}}$ over the common sampling period $T$ are described by the time-invariant system

$$
\begin{gathered}
\hat{S}^{T}: \xi(l+1)=\Phi \xi(l) \\
y(l)=\Psi \xi(l)
\end{gathered}
$$

where we use the notation $\xi(l) \equiv \xi(l, 0), y(l) \equiv y(l, 0), \Psi=\Psi(0)=$ $\left[\begin{array}{lll}C & 0 & 0\end{array}\right]$, and compute $\Phi$ as

$$
\Phi=\Phi(M-1) \cdots \Phi(1) \Phi(0) .
$$

The decentralized control problem is defined as that of choosing the parameters $F_{i}, G_{i}, H_{i}, K_{i}$ of the controllers $C_{i}, i=1,2, \cdots, \nu$ such that the closed-loop system $\hat{S}^{T}$ has desired properties. A solution of this problem is obtained by first making the system $\boldsymbol{S}$ reachable and observable through a single channel by using static feedback of the form (2.15) in all other channels. Then, a dynamic digital controller $C_{i}$ of the form (2.13) is used to achieve a satisfactory performance of $\hat{S}^{T}$. The strategy does not rule out use of dynamic controllers $C_{i}$ in any of the channels. This is a well-known approach in continuous-time decentralized control (Corfmat and Morse [9], Anderson and Moore [12]).

\section{Decentralized Control}

We start with the case of two-channel systems where we make a crucial assumption that the sampling period in one of the channels is an integral multiple of the sampling period in the other channel. Without loss of generality, we set $M_{1}=M$ and $M_{2}=1$ so that $T_{1}=T=M \tau$ and $T_{2}=\tau$. Following the announced control strategy, we apply a static feedback

$$
u_{2}(l, m)=K_{2} y_{2}(l, m)
$$

to channel two to get

$$
\begin{gathered}
S_{1}^{t}: \xi_{1}(l, m+1)=\Phi_{1}(m) \xi_{1}(l, m)+\Gamma_{1}(m) u_{1}(l, m) \\
y_{1}(l, m)=\Psi_{1}(m) \xi_{1}(l, m)
\end{gathered}
$$

where $\xi_{1}(l, m)=\left[x^{T}(l, m), v_{1}^{T}(l, m), v_{2}^{T}(l, m)\right]^{T}$, and

$$
\Psi_{1}(m)=\left[\begin{array}{ccc}
A+B_{2} K_{2} C_{2} & {\left[1-\Delta_{1}(m)\right] B_{1}} & 0 \\
0 & {\left[1-\Delta_{1}(m)\right] I} & 0 \\
K_{2} C_{2} & 0 & 0
\end{array}\right] \text {, }
$$

$\Gamma_{1}(m)=\left[\begin{array}{c}\Delta_{1}(m) B_{1} \\ \Delta_{1}(m) I \\ 0\end{array}\right]$

$\Phi_{1}(m)=\left[\begin{array}{lll}\Delta_{1}(m) C_{1} & 0 & 0\end{array}\right]$.

As before, the transition of $S_{1}^{t}$ over the common sampling period $T$ is described as

$$
\begin{gathered}
S_{1}^{T}: \xi_{1}(l+1)=\Psi_{1} \xi_{1}(l)+\Gamma_{1} u_{1}(l) \\
y_{1}(l)=\Phi_{1} \xi_{1}(l)
\end{gathered}
$$

where the constant matrices $\Phi_{1}, \Gamma_{1}$, and $\Psi_{1}$ are computed from (3.3) as

$$
\begin{gathered}
\Phi_{1}=\Phi_{1}(M-1) \cdots \Phi_{1}(1) \Phi_{1}(0)=\left[\begin{array}{ccc}
\tilde{\Phi}_{2}^{M} & 0 & 0 \\
0 & 0 & 0 \\
K_{2} C_{2} \tilde{\Phi}_{2}^{M-1} & 0 & 0
\end{array}\right] \\
\Gamma_{1}=\Phi_{1}(M-1) \cdots \Phi_{1}(1) \Gamma_{1}(0)=\left[\begin{array}{c}
P_{1}\left(\tilde{\Phi}_{2}\right) B_{1} \\
K_{2} C_{2} P_{1}\left(\tilde{\Phi}_{2}\right) B_{1}
\end{array}\right] \\
\Psi_{1}=\Psi_{1}(0)=\left[\begin{array}{lll}
C_{1} & 0 & 0
\end{array}\right]
\end{gathered}
$$

and

$$
\begin{gathered}
\tilde{\Phi}_{2}=A+B_{2} K_{2} C_{2} \\
P_{1}\left(\tilde{\Phi}_{2}\right)=\tilde{\Phi}_{2}^{M-1}+\cdots+\tilde{\Phi}_{2}+I .
\end{gathered}
$$

We observe from $\Phi_{1}$ and $\Psi_{1}$ that the states $v_{1}(l)$ and $v_{2}(l)$ of the hold circuits are unobservable from $y_{1}(l)$. Since these states are associated with modes at the origin, they can safely be omitted from $S_{1}^{T}$ to obtain a reduced-order system

$$
\begin{gathered}
\tilde{S}_{1}^{T}: x(l+1)=\tilde{\Phi}_{1} x(l)+\tilde{\Gamma}_{1} u_{1}(l) \\
y_{1}(l)=C_{1} x(l)
\end{gathered}
$$

where

$$
\tilde{\Phi}_{1}=\tilde{\Phi}_{2}^{M}, \tilde{\Gamma}_{1}=P_{1}\left(\tilde{\Phi}_{2}\right) B_{1} .
$$

Our purpose is to derive conditions under which the system $\tilde{S}_{1}^{T}$ represented by the triple $\left(\tilde{\Phi}_{1}, \tilde{\Gamma}_{1}, C_{1}\right)$ can be made reachable and observable by a proper choice of the feedback gain $K_{2}$ in (3.1). For this, we first state the following.

(3.9) Lemma: Suppose that a triple $\left(\tilde{\Phi}_{2}, B_{1}, C_{1}\right)$ is reachable and observable, and that $\tilde{\Phi}_{2}$ has no eigenvalues of the form $\rho \exp$ $(j 2 k \pi / M), \rho \geq 0, k=0,1, \cdots, M-1$. Then, $\tilde{\boldsymbol{S}}_{1}^{T}$ is reachable and observable.

Proof: Let the distinct eigenvalues of $\tilde{\Phi}_{2}$ be denoted by $\mu_{i}, i=$ $1,2, \cdots, n_{2}, n_{2} \leq n$. We define a polynomial

$$
q(z, \mu)=z^{M-1}+\mu z^{M-2}+\cdots+\mu^{M-2} z+\mu^{M-1} .
$$

By assumption on $\mu_{i}$, we have

$$
q\left(\mu_{i}, \mu_{i}\right)=M \mu_{i}^{M-1} \neq 0
$$

$$
\begin{gathered}
q\left(\mu_{i}, \mu_{j}\right)=\frac{\mu_{i}^{M}-\mu_{j}^{M}}{\mu_{i}-\mu_{j}} \neq 0, \quad \mu_{i} \neq \mu_{j} \\
q\left(1, \mu_{j}\right)=\frac{1-\mu_{j}^{M}}{1-\mu_{j}} \neq 0
\end{gathered}
$$


for $i, j=1,2, \cdots, n_{2}$, so that the matrices

$$
\begin{gathered}
Q_{i}\left(\tilde{\Phi}_{2}\right)=q\left(\mu_{i}, \tilde{\Phi}_{2}\right), \quad i=1,2, \cdots, n_{2} \\
P_{1}\left(\tilde{\Phi}_{2}\right)=q\left(1, \tilde{\Phi}_{2}\right)
\end{gathered}
$$

are nonsingular.

If the pair $\left(\tilde{\Phi}_{1}, \tilde{\Gamma}_{1}\right)$ is not reachable, then for some $\mu_{i}$

$$
\operatorname{rank}\left[\tilde{\Phi}_{2}^{M}-\mu_{i}^{M} I, \tilde{\Gamma}_{1}\right]<n
$$

Noting that $\tilde{\Phi}_{2}^{M}-\mu_{i}^{M} I=Q_{i}\left(\tilde{\Phi}_{2}\right)\left(\tilde{\Phi}_{2}-\mu_{i} I\right)$, and $\tilde{\Gamma}_{1}=P_{1}\left(\tilde{\Phi}_{2}\right) B_{1},(3.13)$ implies that there exists an $n$ vector $p \neq 0$ such that

$$
p^{T} Q_{i}\left(\tilde{\Phi}_{2}\right)\left(\tilde{\Phi}_{2}-\mu_{i} I\right)=0, \quad p^{T} P_{1}\left(\tilde{\Phi}_{2}\right) B_{1}=0 .
$$

Defining an $n$ vector $r \neq 0$ as $r^{T}=p^{T} P_{1}\left(\tilde{\Phi}_{2}\right)$, and observing that the matrices $P_{1}^{-1}\left(\tilde{\Phi}_{2}\right), Q_{i}\left(\tilde{\Phi}_{2}\right)$, and $\left(\tilde{\Phi}_{2}-\mu_{i} I\right)$ commute, we get from (3.14),

$$
r^{T}\left[\tilde{\Phi}_{2}-\mu_{i} I, B_{1}\right]=0 .
$$

This, however, contradicts the assumption that $\left(\tilde{\Phi}_{2}, B_{1}\right)$ is reachable Therefore, the pair $\left(\tilde{\Phi}_{1}, \tilde{\Gamma}_{1}\right)$ is reachable. Observability of the pair $\left(\tilde{\Phi}_{1}, C_{1}\right)$ can be proved likewise. Q.E.D.

The following result provides conditions for the existence of a proper choice of the feedback gain $K_{2}$

(3.16) Lemma: The system $\tilde{S}_{1}^{T}$ is reachable and observable for almost all $K_{2}$ if the following conditions are satisfied.

(i) The triple $\left(A,\left[B_{1}, B_{2}\right],\left[C_{1}^{T}, C_{2}^{T}\right]^{T}\right)$ is reachable and observable.

(ii) $C_{1}(z I-A)^{-1} B_{2} \not \equiv 0, C_{2}(z I-A)^{-1} B_{1} \not \equiv 0$.

(iii) $\operatorname{rank}\left[\begin{array}{cc}z I-A & B_{1} \\ C_{2} & 0\end{array}\right] \geq n, \operatorname{rank}\left[\begin{array}{cc}z I-A & B_{2} \\ C_{1} & 0\end{array}\right] \geq n$ for all $z$.

(iv) The matrix $A$ has no eigenvalues of the form $\rho \exp (j 2 k \pi / M), \rho \geq$ $0, k=0,1, \cdots, M-1$.

Proof: Assumptions (i)-(iii) guarantee that the triple $\left(\tilde{\Phi}_{2}, B_{1}, C_{1}\right)$ is reachable and observable for almost all $K_{2}$ (Corfmat and Morse [9]). Assumption (iv) together with the fact that the eigenvalues of $\tilde{\Phi}_{2}$ depend continuously on $K_{2}$ ensure that $\tilde{\Phi}_{2}$ has no eigenvalues of the form in (iv) for almost all $K_{2}$. Then, the proof follows from Lemma (3.9). Q.E.D.

Except for condition (iv), Lemma (3.16) represents a single-rate discrete version of the well-known result for decentralized control of continuous-time systems (Corfmat and Morse [9]). The multirate feature is captured by Lemma (3.9).

Finally, we reformulate the obtained conditions of Lemmas (3.9) and (3.16) in terms of the original continuous-time plant $\boldsymbol{P}$.

(3.I7) Lemma: Suppose that a continuous-time plant $\boldsymbol{P}$ with a triple $\left(\bar{A},\left[\bar{B}_{1}, \bar{B}_{2}\right],\left[C_{1}^{T}, C_{2}^{T}\right]^{T}\right)$ satisfies conditions (i)-(iii) of Lemma (3.16). Then, for almost any common sampling period $T$, the single-rate discrete model defined by the triple $\left(A,\left[B_{1}, B_{2}\right],\left[C_{1}^{T}, C_{2}^{T}\right]^{T}\right)$ satisfies conditions (i)-(iv) of Lemma (3.16)

Proof: Let the eigenvalues of $\bar{A}$ be $\lambda_{i}=\sigma_{i}+j \omega_{i}, i=1,2, \cdots, n$. Then $A$ satisfies condition (iv) provided

$$
\tau \neq 2(l M+m) \pi / M \omega_{i}, \quad m=0,1, \cdots, M-1 ; l=0,1, \cdots .
$$

This condition also guarantees that there are no hidden oscillations in the single-rate discrete model, that is, $\left(A,\left[B_{1}, B_{2}\right],\left[C_{1}^{T}, C_{2}^{T}\right]^{T}\right)$ satisfies condition (i), which, in turn, implies condition (ii).

To show that the discrete model satisfies the remaining condition (iii), we consider a particular case where $\bar{A}$ has only distinct eigenvalues $\lambda_{i}, i=1,2, \cdots, n$, and the plant $\boldsymbol{P}$ has single-input-single-output channels, that is, $p_{i}=q_{i}=1, i=1,2$. The proof for the general case is not essentially different, but involves technicalities and is, therefore, omitted. For the particular case, assume without loss of generality that $\bar{A}=\operatorname{diag}\left\{\lambda_{1}, \lambda_{2}, \cdots, \lambda_{n}\right\}$, and let $\bar{B}_{1}=\left(\bar{b}_{1}, \bar{b}_{2}, \cdots, \bar{b}_{n}\right)^{T}, C_{2}=$ $\left(c_{1}, c_{2}, \cdots, c_{n}\right)$. Then, $A=\operatorname{diag}\left\{\mu_{1}, \mu_{2}, \cdots, \mu_{n}\right\}$ where $\mu_{i}=\exp \left(\lambda_{i} \tau\right)$, and $B=\left(b_{1}, b_{2}, \cdots, b_{n}\right)^{T}$ where $b_{i}=\int_{0}^{\tau} \exp \left(\lambda_{i} t\right) \bar{b}_{i} d t$. We claim that for almost all $\tau$,

$\operatorname{rank}\left[\begin{array}{cc}A-\mu_{i} I & B_{1} \\ C_{2} & 0\end{array}\right]$

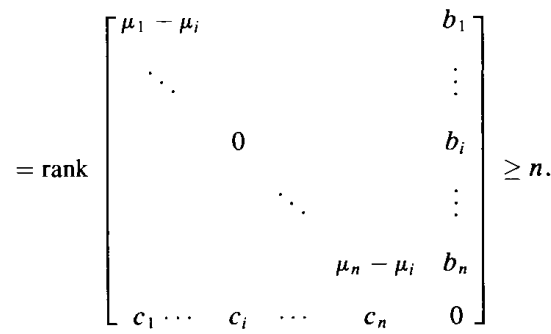

To prove the claim, suppose (3.19) is violated for some fixed $i$. Then we must have $b_{i}=c_{i}=0$ and $\gamma_{i}(\tau)=0$ where $\gamma_{i}(\tau)$ is the determinant of the $n \times n$ matrix obtained by deleting the $i$ th row and the $i$ th column from the matrix in (3.19). By induction on the order of the resulting submatrix, it is straightforward to show that

$$
\gamma_{i}^{(n-1)}(0)=(n-1) ! \bar{\gamma}_{i}
$$

where superscript $(n-1)$ indicates the $(n-1)$ st derivative and $\bar{\gamma}_{i}$ is the determinant of the $n \times n$ matrix obtained by deleting the $i$ th row and $i$ th column of the matrix

$$
\left[\begin{array}{cc}
\bar{A}-\lambda_{i} I & \bar{B}_{1} \\
C_{2} & 0
\end{array}\right]
$$

By assumption (iii) concerning the continuous plant $P, \bar{\gamma}_{i} \neq 0$ if $\bar{b}_{i}=$ $c_{i}=0, i=1,2, \cdots, n$. Since $\gamma_{i}(\tau)$ is an analytic function, $\bar{\gamma}_{i} \neq 0$ implies by (3.20) that $\gamma_{i}(\tau)$ is zero for at most a finite number of values of $\tau$ in every finite interval. Therefore, (3.19) holds for all $i=1,2, \cdots, n$, and almost all $\tau$. This proves the first inequality in (iii) of Lemma (3.16). By replacing $B_{1}$ by $B_{2}$ and $C_{1}$ by $C_{2}$, the second inequality follows.

Q.E.D.

Finally, by combining Lemmas (3.9), (3.16), and (3.17), we arrive at our main result which we state as a theorem, the proof of which is automatic.

(3.22) Theorem: If a continuous-time plant $\boldsymbol{P}$ satisfies the conditions (i)-(iii) of Lemma (3.16), then the corresponding multirate sampled-data system $\tilde{S}_{1}^{T}$ is reachable and observable for almost any common sampling period $T$ and almost any feedback gain $K_{2}$.

This is a welcome result in the context of decentralized control because it allows for choosing different sampling rates in the individual channels to fit the corresponding characteristic frequencies, without a serious concern about the consequences of the multirate operation in selecting appropriate digital controllers to meet a desired performance of the overall system.

\section{Further Results}

In this section we consider some variations and extensions of the results obtained in the previous section.

\section{A. Use of Dynamic Digital Controllers}

We first investigate the effect of replacing the static feedback in the second channel of the two-channel system considered in Section III by a digital controller. Applying a dynamic feedback of the form (2.14) instead of the static feedback of (3.1), we obtain an extended system

$$
\begin{gathered}
S_{1 e}: \xi_{1 e}(l, m+1)=\Phi_{1 e}(m) \xi_{1 e}(l, m)+\Gamma_{1 e}(m) u_{1}(l, m) \\
y_{1}(l, m)=\Psi_{1 e}(m) \xi_{1 e}(l, m)
\end{gathered}
$$


where $\xi_{1 e}(l, m)=\left[x^{T}(l, m), z_{2}^{T}(l, m), v_{1}^{T}(l, m), v_{2}^{T}(l, m)\right]^{T}$, and

$\Phi_{1 e}(m)=\left[\begin{array}{cccc}A+B_{2} K_{2} C_{2} & B_{2} H_{2} & {\left[1-\Delta_{1}(m)\right] B_{1}} & 0 \\ G_{2} C_{2} & F_{2} & 0 & 0 \\ 0 & 0 & {\left[1-\Delta_{1}(m) I\right]} & 0 \\ K_{2} C_{2} & H_{2} & 0 & 0\end{array}\right]$,

$$
\Gamma_{l e}(m)=\left[\begin{array}{c}
\Delta_{1}(m) B_{1} \\
0 \\
\Delta_{1}(m) I \\
0
\end{array}\right]
$$$$
\Psi_{1 e}(m)=\left[\begin{array}{lll}
\Delta_{1}(m) C_{1} & 0 & 0
\end{array}\right] .
$$

Defining the matrices

$$
\begin{gathered}
A_{e}=\left[\begin{array}{cc}
A & 0 \\
0 & F_{2}^{\prime}
\end{array}\right], B_{1 e}=\left[\begin{array}{c}
B_{1} \\
0
\end{array}\right], B_{2 e}=\left[\begin{array}{cc}
B_{2} & 0 \\
0 & I
\end{array}\right] \\
C_{1 e}=\left[\begin{array}{ll}
C_{1} & 0
\end{array}\right] \\
C_{2 e}=\left[\begin{array}{cc}
C_{2} & 0 \\
0 & I
\end{array}\right], K_{2 e}=\left[\begin{array}{cc}
K_{2} & H_{2} \\
G_{2} & F_{2}-F_{2}^{\prime}
\end{array}\right]
\end{gathered}
$$

where $F_{2}^{\prime}$ is an arbitrary matrix having no eigenvalues of the form $\rho \exp$ $(j 2 k \pi / M)$, we observe that $\Phi_{1 e}(m), \Gamma_{1 e}(m)$, and $\Psi_{l e}$ of $(4.2)$ are of the same type as $\Phi_{1}(m), \Gamma_{1}(m)$, and $\Phi_{1}$ of (3.3) except that the matrices $A, B_{1}, \cdots$, etc., are replaced by $A_{e}, B_{1 e}, \cdots$, etc. Therefore, following the same steps as in the previous section, we obtain a time-invariant system $S_{1 e}^{T}$ describing the transitions of $S_{1 e}^{\tau}$ over the common sampling period, which is then reduced to

$$
\begin{aligned}
\tilde{S}_{1 e}^{T}: x_{e}(l+1) & =\tilde{\Phi}_{1 e} x_{e}(l)+\tilde{\Gamma}_{1 e} u_{1}(l) \\
y_{1}(l) & =C_{1 e} x_{e}(l)
\end{aligned}
$$

where $x_{e}(l)=\left[x^{T}(l), z_{2}^{T}(l)\right]^{T}$, and

$$
\tilde{\Phi}_{1 e}=\tilde{\Phi}_{2 e}^{M}, \tilde{\Gamma}_{l e}=P_{1}\left(\tilde{\Phi}_{1 e}\right) B_{1 e}
$$

with

$$
\tilde{\Phi}_{2 e}=A_{e}+B_{2 e} K_{2 e} C_{2 e}=\left[\begin{array}{cc}
A+B_{2} K_{2} C_{2} & B_{2} H_{2} \\
G_{2} C_{2} & F_{2}
\end{array}\right] .
$$

A modification of Theorem (3.22), which accommodates a dynamic controller $C_{2}$ in channel two, is given as follows.

(4.7) Theorem: Under the conditions of Theorem (3.22), the multirate sampled-data system $\tilde{\boldsymbol{S}}_{1 e}^{T}$ is reachable and observable for almost any common sampling period $\tau$ and almost any digital controller $C_{2}$ represented by the quadruple $\left(F_{2}, G_{2}, H_{2}, K_{2}\right)$.

Proof: We first note that both Lemma (3.9) and Lemma (3.16) remain valid when the matrices $A, \check{\Phi}_{2}, B_{1}, B_{2}, C_{1}, C_{2}, K_{2}$, and the system $S_{1}^{T}$ are replaced by the matrices $A_{e}, \tilde{\Phi}_{2 e}, B_{1 e}, B_{2 e}, C_{1 e}$, $C_{2 e}, K_{2 e}$, and the system $\tilde{S}_{1 e}^{T}$. On the other hand, if the triple $\left(A,\left[B_{1}, B_{2}\right],\left[C_{1}^{T}, C_{2}^{T}\right]^{T}\right)$ satisfies conditions (i)-(iv) of Lemma (3.16), then so does the triple $\left(A_{e},\left[B_{1 e}, B_{2 e}\right],\left[C_{1 e}^{T}, C_{2 e}^{T}\right]^{T}\right)$. Therefore, the conclusion of Lemma (3.17) also holds for the latter triple. Q.E.D.

\section{B. $\nu$-Channel Systems}

We next consider a three-channel system in an attempt to extend the results of Section III to the general case of $\nu$-channel systems. As in Section III, we restrict our attention to the case where the sampling periods of the channels are integral multiples of one another, and assume, without loss of generality, that $M_{3}=1, M_{2}=N_{2}$, and $M_{1}=M=$ $N_{1} N_{2}$, that is, $T_{3}=\tau, T_{2}=N_{2} \tau$, and $T_{1}=T=N_{1} N_{2} \tau$.

When the third channel is closed by a static feedback matrix $K_{3}$, the transitions of the resulting two-channel discrete-time reduced-order system over the sampling period $T_{2}=N_{2} \tau$ are represented by the triple $\tilde{\boldsymbol{S}}_{12}^{T_{2}}=\left\{\tilde{\Phi}_{3}^{N_{2}},\left[\boldsymbol{P}_{2}\left(\tilde{\Phi}_{3}\right) B_{1}, P_{2}\left(\tilde{\Phi}_{3}\right) B_{2}\right],\left[C_{1}^{T}, C_{2}^{T}\right]^{T}\right\}$ where $\tilde{\Phi}_{3}=$ $A+B_{3} K_{3} C_{3}$ and $P_{2}\left(\tilde{\Phi}_{3}\right)=\tilde{\Phi}_{3}^{N_{2}-1}+\cdots+\tilde{\Phi}_{3}+I$. When, in addition, the second channel is closed by a feedback matrix $K_{2}$, we obtain a single-channel system $\tilde{S}_{1}^{T}=\left\{\tilde{\Phi}_{1}^{N_{1}}, P_{1}\left(\tilde{\Phi}_{2}\right) P_{2}\left(\tilde{\Phi}_{3}\right) B_{1}, C_{1}\right\}$ with $\tilde{\Phi}_{2}=\tilde{\Phi}_{3}^{N_{2}}+P_{2}\left(\tilde{\Phi}_{3}\right) B_{2} K_{2} C_{2}$, which represents the transitions over the common sampling period $T$.

Obviously, after the feedback loop in channel three is closed, the decentralized control problem is reduced to that of a two-channel system, and thus can be resolved using the results of Section III provided the two-channel system $\tilde{S}_{12}^{T_{2}}$ satisfies the conditions of Lemma (3.16). From the knowledge about decentralized control of continuoustime systems, it is easy to see that this is possible with a proper choice of $K_{3}$ only if all the complementary subsystems of the triple $\left(A,\left[B_{1}, B_{2}, B_{3}\right],\left[C_{1}^{T}, C_{2}^{T}, C_{3}^{T}\right]^{T}\right)$ are complete [9]. Unlike continuoustime systems, completeness of the complementary subsystems of the latter triple is not generally sufficient for the former triple to satisfy the conditions of Lemma (3.16). Obviously, the difficulty arises from the multirate nature of the system. Fortunately, this difficulty can be overcome by choosing a slightly different common sampling period. With this in mind, we state conditions for a $\nu$-channel system to be made reachable and observable from the first channel in terms of the continuous-time plant $P$.

(4.8) Theorem: Suppose that a $\nu$-channel continuous-time plant $P$ is centrally reachable and observable, and that every complementary subsystem of $\boldsymbol{P}$ is complete. Then, the multirate sampled-data system $\tilde{\boldsymbol{S}}_{1}^{T}$, which is obtained by closing all channels except the first, is reachable and observable for almost any common sampling period $T$ and almost any feedback gains $K_{2}, K_{3}, \cdots, K_{y}$.

\section{Rational Ratios of Sampling Periods}

Our final comment concerns the case when the ratio $T_{1} / T_{2}$ is a rational number rather than an integer. In this case, the transitions of the system over the sampling period $T_{1}$ after the second channel is closed using static feedback are no longer described by a time-invariant system, but rather by a periodically varying system with the period $M / M_{1}$. An obvious way to control this system from the first channel is to apply a control $u_{1}\left(m_{1}\right)$ which is constant throughout the common sampling period $T$. The net effect of this strategy is to replace the periodically time-varying system by a time-invariant system whose transitions are described at the common sampling periods. Then, the results of Section III apply directly, but the controller $C_{2}$ may have to be time varying. This simple strategy is neither unique nor best, and freedom in changing $u_{1}\left(m_{1}\right)$ at the sampling irstants corresponding to $T_{1}$ can be exploited to obtain a suitable time-invariant controller $C_{2}$.

\section{Conclusion}

Our main result is that the decentralized stabilizability conditions for a continuous-time plant are all one needs to control the plant by applying multirate digital controllers. This is encouraging and not entirely expected. It opens up a possibility to try other control strategies which were successful for continuous-time systems. In particular, one can attempt a generalization of the notion of fixed modes, explore the possibility of using time-varying feedback, and formulate decentralized multirate optimization problems. An attractive aspect of the proposed scheme is that the solutions of these problems can be implemented using parallel processing.

\section{ReFERENCES}

[1] J. Sklansky and J. R. Ragazzini, "Analysis of errors in sampled-data feedback systems," AIEE Trans., vol. 74, pp. 65-71, 1955.

[2] G. Kranc, "Compensation of an error-sampled system by a multi-rate controller," AIEE Trans., vol. 76, pp. 149-159, 1957.

[3] R. E. Kalman and J. Bertram, "A unified approach to the theory of sampling systems," J. Franklin Inst., vol. 267, pp. 405-435, 1959.

[4] C. H. Lu and S. C. Gupta, "Multi-rate digital filtering," Int. J. Syst. Sci., vol. 10, pp. 605-620, 1979.

[5] D. P. Glasson, "Development and application of multi-rate digital control," IEEE Contr. Syst. Mag., vol. 3, pp. 2-8, 1983.

[6] M. Araki and K. Yamamoto, "Multivariable multirate sampled-data systems: State-space description, transfer characteristics, and Nyquist criterion," IEEE
IEnstems: Trans. Automat. Contr., vol. AC-31, pp. 145-154, 1986. 
[7] M. Araki and T. Hagiwara, "Pole assignment by multirate sampled-data output feedback," Int. J. Contr., vol. 44, pp. 1661-1673, 1986.

[8] S. H. Wang and E. J. Davison, "On the stabilization of decentralized control systems," IEEE Trans. Automat. Contr., vol. AC-18, pp. 473-478, 1973.

[9] J. P. Corfmat and A. S. Morse, "Decentralized control of linear multivariable system," Automatica, vol. 12, pp. 479-495, 1976.

[10] M. E. Sezer and D. D. Siljak, "Structurally fixed modes," Syst. Contr. Lett., vol. 1, pp. 60-64, 1981 .

[11] N. Amit, "Optimal control of multirate digital control systems," Stanford Univ. Stanford, CA, Rep. SUDAAR 523, 1980.

[12] B. D. O. Anderson and J. B. Moore, "Time-varying feedback laws for decentralized control," IEEE Trans. Automat. Contr., vol. AC-26, pp. 1133-1139, 1981.

\section{On Methods of Treating dc Levels in an Adaptive Digital Smith Predictor}

\author{
C. C. HANG AND B. W. CHONG
}

\begin{abstract}
Two popular methods of treating unknown or slowly varying dc levels in the input and output measurements used for parameter estimation in an adaptive digital Smith predictor are studied. They are the "high-pass filtering" method and the method of "estimating an additional constant." The performance of the former is found to be inferior in that it leads to a larger output variance under stochastic environment. This is caused by the higher variation in model output due to static gain variations in the estimated explicit model.
\end{abstract}

\section{Introduction}

The digital Smith predictor [1]-[4] is a popular model-based approach to the control of systems with long dead time. The feasibility of an adap tive Smith predictor using on-line parameter estimation has already been demonstrated [3], [4]. Attention has now been switched to the practical implementation aspects.

One such practical problem in parameter estimation for adaptive control is the presence of dc levels in the input and output measurements. These unknown or slowly varying dc values are difficult to estimate on line and several solutions have been proposed [1], the most popular of which are "high-pass filtering" and "estimation of a dc constant." These two methods have been found to be comparable when used with most of the self-tuning controllers with integral action [5]. However, it will be shown in this paper that this need not be the same when they are applied to the adaptive digital Smith predictor which uses an explicit model in the control algorithm.

\section{Adaptive Digital Smith Predictor}

The digital Smith predictor [4] is shown in Fig. 1. The block $M\left(z^{-1}\right)$ represents the plant dynamics without the dead time. The block $D\left(z^{-1}\right)$ represents the pure/apparent dead time of the process and could include terms caused by a fractional delay [2]. $K_{D}\left(z^{-1}\right)$ is the main controller designed on the basis of the explicit model $M\left(z^{-1}\right)$.

The explicit self-tuning regulator approach [1], [6] is used to implement the adaptation loop in the adaptive digital Smith predictor. We shall limit the following development by using the well-known recursive leas square (RLS) scheme for on-line parameter estimation, which is sufficient in the case of low measurement noise. For simplicity without loss of generality, we shall assume that the plant is described by the following second-order difference equation:

$$
y(k)=\boldsymbol{x}^{T}(k) \theta+\epsilon(k)
$$

Manuscript received February 16, 1988; revised November 28, 1988 The authors are with the Department of Electrical Engineering, National University of Singapore, Singapore 0511 .

IEEE Log Number 8931408

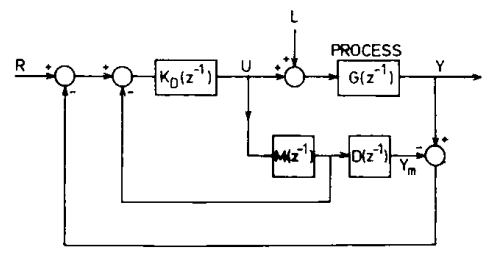

Fig. 1. Block diagram of a digital Smith predictor.

where

$$
\begin{aligned}
& \text { data vector: } \boldsymbol{x}(k)=[-y(k-1)-y(k-2) \\
& u(k-d-1) u(k-d-2)]^{T}
\end{aligned}
$$

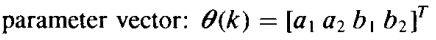

$$
\begin{aligned}
& \text { input change: } u(k)=U(k)-U_{\mathrm{dc}} \\
& \text { output change: } y(k)=Y(k)-Y_{\mathrm{dc}} \\
& \epsilon(k)=\text { white noise } \\
& d=\text { integer dead time in number } \\
& \text { of sampling intervals. }
\end{aligned}
$$

Following the standard RLS formulation [1], [6], we have

$$
\begin{aligned}
\hat{\boldsymbol{\theta}}(k) & =\hat{\boldsymbol{\theta}}(k-1)+\boldsymbol{G}(k) \boldsymbol{e}(k) \\
\boldsymbol{G}(k) & =\left[\boldsymbol{x}^{T}(k) \boldsymbol{P}(k-1) \boldsymbol{x}(k)+\lambda\right]^{-1} \boldsymbol{P}(k-1) \boldsymbol{x}(k) \\
\boldsymbol{P}(k) & =\left[\boldsymbol{P}(k-1)-\boldsymbol{G}(k) \boldsymbol{x}^{T}(k) \boldsymbol{P}(k-1)\right] / \lambda
\end{aligned}
$$

where

$\hat{\boldsymbol{\theta}}(k)=$ estimated parameter vector

$\lambda=$ scalar forgetting factor

$e(k)=$ prediction error.

In practice, the input and output measurements $U(k)$ and $Y(k)$ contain unknown dc values $U_{\mathrm{dc}}$ and $Y_{\mathrm{dc}}$ which are difficult to estimate separately in on-line adaptive control. The two most popular solutions to this problem [1] are outlined in the following.

\section{dc Method One: High-Pass Filtering}

In "high-pass filtering," both the input and output signals are filtered to remove the de levels as follows:

$$
\begin{aligned}
\text { filtered output: } y^{*}(k) & =F_{H}\left(z^{-1}\right) y(k)=F_{H}\left(z^{-1}\right) Y(k) \\
\text { filtered input: } u^{*}(k) & =F_{H}\left(z^{-1}\right) u(k)=F_{H}\left(z^{-1}\right) U(k) \\
F_{H}\left(z^{-1}\right) & =\frac{\beta\left(1-z^{-1}\right)}{1-\alpha z^{-1}} .
\end{aligned}
$$

Hence,

$$
\begin{aligned}
e(k) & =y^{*}-\hat{y}^{*} \\
\hat{y}^{*} & =\boldsymbol{x}^{T}(k) \hat{\boldsymbol{\theta}}(k-1) \\
\boldsymbol{x}(k) & =\left[-y^{*}(k-1)-y^{*}(k-2) u^{*}(k-d-1) u^{*}(k-d-2)\right]^{T} \\
\hat{\boldsymbol{\theta}}(k) & =\left[\hat{a}_{1} \hat{a}_{2} \hat{b}_{1} \hat{b}_{2}\right]^{T} .
\end{aligned}
$$

The solution is thus simply to replace the data vector in (2) by that in (5).

\section{dc Method Two: Estimation of a dc Constant}

In this case, we estimate the effect of $U_{\mathrm{dc}}$ and $Y_{\mathrm{dc}}$ indirectly so that 